\title{
A LATTICE STRUCTURE OF BIORTHOGONAL LINEAR-PHASE FILTER BANKS WITH HIGHER-ORDER FEASIBLE BUILDING BLOCKS
}

\author{
Yuichi Tanaka ${ }^{1}$, Masaaki Ikehara ${ }^{1}$, and Truong Q. Nguyen ${ }^{2}$ \\ 1: EEE Dept., Keio University \\ Yokohama, Kanagawa, 223-8522 Japan \\ e-mail: \{ytanaka, ikehara\}@ tkhm.elec.keio.ac.jp \\ 2: ECE Dept., University of California, San Diego \\ La Jolla, CA, 92093 USA \\ e-mail: nguyent@ece.ucsd.edu
}

\section{ABSTRACT}

This paper proposes a new structure of biorthogonal linear-phase filter banks (BOLPFBs) by using building blocks which can be realized long filters with fewer number of building blocks than conventional ones. The structure is derived from a generalization of the building blocks of first-order LPFBs. The resulting FBs have good performance in stopband attenuation and low implementation costs.

\section{INTRODUCTION}

Filter banks (FBs) and their applications in the wide area of signal processing have been studied for a few decades [1]. There are many properties depending on the requirements. In practical applications, linear-phase (LP) property is highly desirable since the symmetric extension can be used at signal boundaries. Moreover, perfect reconstruction (PR) is one of the most important properties especially in the field of signal compression. In this paper, we propose a new structure of LPPRFBs.

One of the most efficient approaches to implement FBs is the lattice structure [1]. It is based on a factorization of polyphase matrices of FBs. If high-order FBs are desired, they can be realized by cascading lower-ordered building blocks. Usually order-1 (the highest order of $z^{-1}$ is one) building blocks are adopted as the lowest order. The cascaded order- 1 structure is effective in the viewpoint of achieving any-order FBs. However, the high-order FB requires many order-1 building blocks which increase the implementation cost.

Therefore, finding the efficient structure of order- $N$ building blocks is an interesting research problem. Generally it is very complicated since a general order- $N$ building block is composed of a matrix polynomial of delay elements $z^{-i}$ (i: some integer). The problem is to guarantee the LP property and calculate its inverse with a reasonable cost for the synthesis bank.

Makur et al. proposed a new type of LPFBs called firstorder (FO) LPFBs [3]. They are generalized versions of $M \times 2 M$ biorthogonal (BO) LPFBs [2] where the synthesis filter lengths can be longer than the analysis ones. In other words, each of them has an order- 1 analysis building block and an order- $N(N \geq 1)$ synthesis building block. It is an attractive feature and helps to derive an efficient order- $N$ structure.

This paper introduces a new building block structure called higher-order feasible (HOF) building block which can be permitted higher-ordered BOLPFBs with fewer building blocks than the cascaded order-1 structure. Furthermore, building blocks of BOLPFBs and FOLPFBs are subclasses of HOF building blocks. The proposed FBs can yield long filters with reasonable implementation costs.

Notations: The identity and reversal matrices are $\mathbf{I}$ and $\mathbf{J}$, respectively. Also, $\operatorname{diag}(\cdot)$ denotes a block diagonal matrix. For simplicity, we omit matrix sizes when they are obvious.
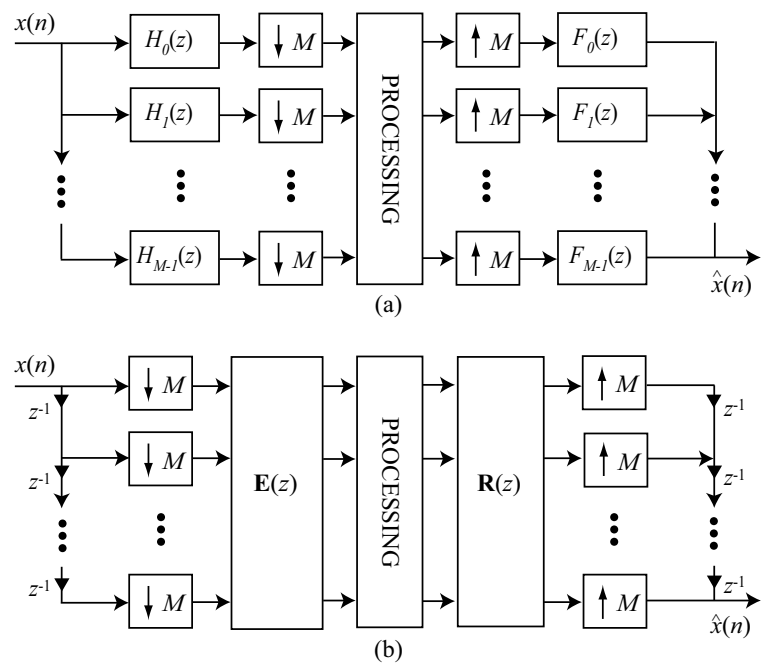

Figure 1: $M$-channel maximally decimated filter bank. (a) conventional representation. (b) polyphase representation.

\section{REVIEW}

\subsection{BOLPFBs}

Consider an $M$-channel BOLPFB with filter length $K M$ [2]. A typical structure of a FB and its polyphase representation are shown in Fig. 1. Using the lattice structure, the analysis polyphase matrix $\mathbf{E}(z)$ can always be represented as

$$
\mathbf{E}(z)=\mathbf{G}_{K-1}(z) \mathbf{G}_{K-2}(z) \ldots \mathbf{G}_{1}(z) \mathbf{E}_{0} .
$$

If $\mathrm{PR}$ is achieved, the causal synthesis polyphase matrix $\mathbf{R}(z)$ is given as

$$
\mathbf{R}(z)=z^{-(K-1)} \mathbf{E}_{0}^{-1} \mathbf{G}_{1}^{-1}(z) \mathbf{G}_{2}^{-1}(z) \ldots \mathbf{G}_{K-1}^{-1}(z) .
$$

When $M$ is even, each matrix in (1) is represented as follows:

$$
\mathbf{G}_{i}(z)=\boldsymbol{\Phi}_{i} \mathbf{W} \boldsymbol{\Lambda}(z) \mathbf{W}, \quad \mathbf{E}_{0}=\frac{1}{\sqrt{2}}\left[\begin{array}{cc}
\mathbf{U}_{0} & \mathbf{U}_{0} \mathbf{J} \\
\mathbf{V}_{0} & -\mathbf{V}_{0} \mathbf{J}
\end{array}\right]
$$

where $\boldsymbol{\Phi}_{i}=\operatorname{diag}\left(\mathbf{U}_{i}, \mathbf{V}_{i}\right)$ and

$$
\mathbf{W}=\frac{1}{\sqrt{2}}\left[\begin{array}{cc}
\mathbf{I}_{M / 2} & \mathbf{I}_{M / 2} \\
\mathbf{I}_{M / 2} & -\mathbf{I}_{M / 2}
\end{array}\right], \quad \boldsymbol{\Lambda}(z)=\left[\begin{array}{cc}
z^{-1} \mathbf{I}_{M / 2} & \mathbf{0}_{M / 2} \\
\mathbf{0}_{M / 2} & \mathbf{I}_{M / 2}
\end{array}\right] .
$$

If the $M / 2 \times M / 2$ matrices $\mathbf{U}_{i}$ and $\mathbf{V}_{i}$ are nonsingular, the $\mathrm{FB}$ is a BOLPFB. Furthermore, $\mathbf{U}_{i}$ for $i>0$ can be set to $\mathbf{U}_{i} \equiv \mathbf{I}$ for simplicity without losing completeness [4]. 


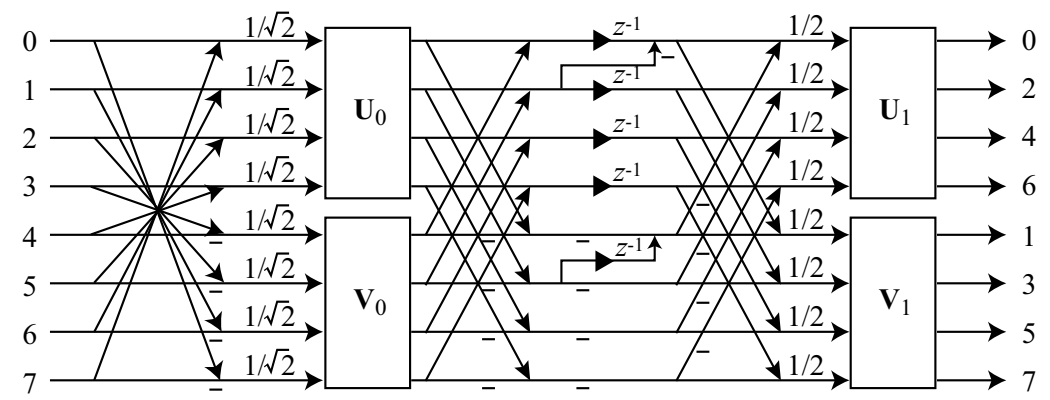

(a)

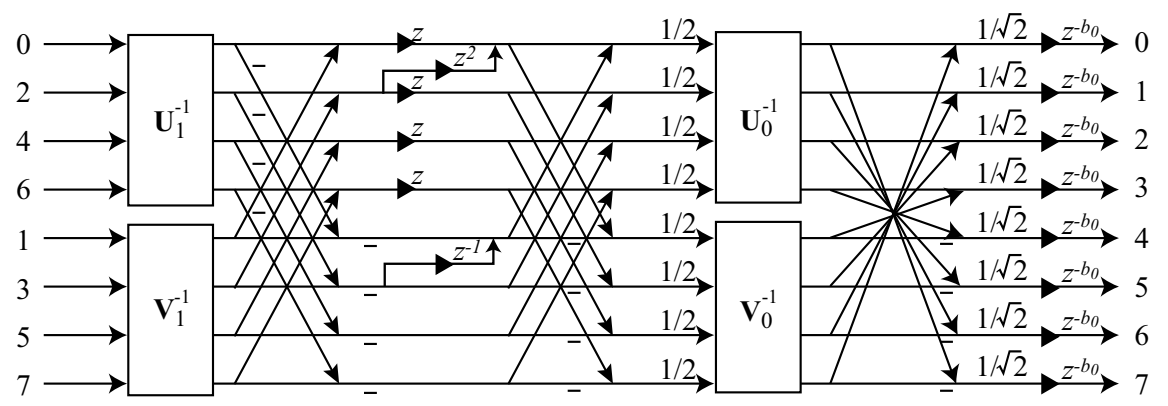

(b)

Figure 2: Lattice structure of a FOLPFB ( $b_{i}=\{2,1,1\}$, shown for $\left.M=8\right)$. (a) analysis bank. (b) synthesis bank.

\subsection{FOLPFBs}

In [3], the eigenstructure based characterization of $M$-channel BOLPFBs whose analysis filter lengths are $2 M$ (they are called firstorder) and synthesis ones are equal to or longer than $2 M$ was presented. Its lattice structure of the analysis bank is

$$
\begin{aligned}
\mathbf{E}(z)= & \operatorname{diag}\left(\mathbf{U}_{1}, \mathbf{V}_{1}\right) \mathbf{W}^{\prime}\left[\begin{array}{cc}
\mathbf{I}_{M / 2} z^{-1}-\mathscr{J} & \mathbf{0}_{M / 2} \\
\mathbf{0}_{M / 2} & \mathscr{J} z^{-1}-\mathbf{I}_{M / 2}
\end{array}\right] \\
& \times \mathbf{W} \operatorname{diag}\left(\mathbf{U}_{0}, \mathbf{V}_{0}\right) \mathbf{W} \operatorname{diag}\left(\mathbf{I}_{M / 2}, \mathbf{J}_{M / 2}\right)
\end{aligned}
$$

where $\mathbf{W}^{\prime}=\frac{1}{\sqrt{2}}\left[\begin{array}{cc}\mathbf{I}_{M / 2} & -\mathbf{I}_{M / 2} \\ \mathbf{I}_{M / 2} & \mathbf{I}_{M / 2}\end{array}\right]$, each $\mathbf{U}_{i}$ and $\mathbf{V}_{i}$ is an $M / 2 \times$ $M / 2$ nonsingular matrix, and $\mathscr{J}$ is an $M / 2 \times M / 2$ block diagonal with Jordan blocks of size $b_{i}\left(i=0, \ldots, n, b_{i}\right.$ is nonincreasing positive integer and $\sum_{i=0}^{n} b_{i}=M / 2$ ) with zero eigenvalue. For example, if $M=6$ and $\left\{b_{i}\right\}=\{2,1\}, \mathscr{J}=\left[\begin{array}{ll|l}0 & 1 & 0 \\ 0 & 0 & 0 \\ \hline 0 & 0 & 0\end{array}\right]$. Also $\mathbf{R}(z)$ is obtained as follows:

$$
\begin{aligned}
\mathbf{R}(z)= & z^{-b_{0}} \operatorname{diag}\left(\mathbf{I}_{M / 2}, \mathbf{J}_{M / 2}\right) \mathbf{W} \operatorname{diag}\left(\mathbf{U}_{0}^{-1}, \mathbf{V}_{0}^{-1}\right) \mathbf{W} \\
& \times\left[\begin{array}{cc}
\mathbf{I}_{M / 2} z+\sum_{i=2}^{b_{0}} \mathscr{J}^{i-1} z^{i} & \mathbf{0}_{M / 2} \\
\mathbf{0}_{M / 2} & -\mathbf{I}_{M / 2}-\sum_{i=1}^{b_{0}-1} \mathscr{J}^{i} z^{-i}
\end{array}\right] \\
& \times \mathbf{W}^{\prime T} \operatorname{diag}\left(\mathbf{U}_{1}^{-1}, \mathbf{V}_{1}^{-1}\right) .
\end{aligned}
$$

In this structure, some patterns of the synthesis filter length can be permitted. If $M=6$, we can design a FOLPFB whose analysis filter length is $2 \times 6=12$ and synthesis length is $12\left(b_{i}=\{1,1,1\}\right)$, $24\left(b_{i}=\{2,1\}\right)$ or $36\left(b_{i}=\{3\}\right)$. Their lattice structure is shown in Fig. 2. For further information of this class of FBs, please refer to the articles $[3,6]$. Obviously, when $b_{i}=\{1, \ldots, 1\}$, the obtained $\mathrm{FB}$ is a BOLPFB.

\section{FORMULATION OF HIGHER-ORDER FEASIBLE BUILDING BLOCKS}

In this section, we introduce the structure and properties of the proposed HOF building blocks. They permit to have various filter lengths with one building block. Furthermore, any-order LPFBs can be designed by cascading the HOF building blocks. To obtain the HOF building blocks, first we generalize the building block of FOLPFBs and then show the complete HOF structure. We consider an even-channel BOLPFB which has the same filter lengths in both analysis and synthesis banks.

\subsection{Third-Order LPFBs as a Generalization of FOLPFBs}

A block-based or time-domain structure of FBs is a good implementation to consider in a signal processing framework [5]. In this paper, the structure helps to realize and understand the proposed HOF building block. The block-based framework at the analysis sides of BOLPFBs and FOLPFBs are depicted in Fig. 3(a) and (b), respectively. For simplicity, both figures are shown for $M=4, K=2$ for the BOLPFB and $b_{i}=\{2\}$ for the FOLPFB. In these figures, $\mathbf{P}_{0}=\mathbf{W} \mathbf{E}_{0}, \mathbf{P}_{1}=\boldsymbol{\Phi}_{1} \mathbf{W}$ and $\mathbf{P}_{2}=\boldsymbol{\Phi}_{1} \mathbf{W}^{\prime}$. It is easily understood that delay elements act as a shift operator at boundaries of each $M \times M$ block transform. The difference between BOLPFBs and FOLPFBs is the existence of lifting operators between two block transforms. To generalize the structure of FOLPFBs, it is natural to focus on the lifting steps.

The typical lifting step for two signals consists of one prediction and one update operators [8]. With this consideration, the matrix with delay elements of FOLPFBs seems to be omitted either prediction or update operator with its coefficients. Conversely, more general structure can be achieved by adding these operators and coefficients. Consequently, the proposed structure with the generalized building block is represented as follows:

$$
\mathbf{E}(z)=\left[\begin{array}{ll}
\mathbf{U}_{1} & \\
& \mathbf{V}_{1}
\end{array}\right] \mathbf{W}^{\prime} \boldsymbol{\Lambda}_{T O}(z) \mathbf{W}\left[\begin{array}{ll}
\mathbf{U}_{0} & \\
& \mathbf{V}_{0}
\end{array}\right] \mathbf{W}\left[\begin{array}{ll}
\mathbf{I} & \\
& \mathbf{J}
\end{array}\right]
$$




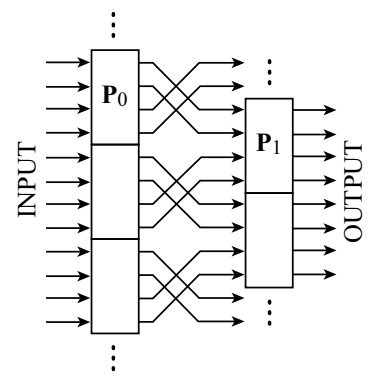

(a)

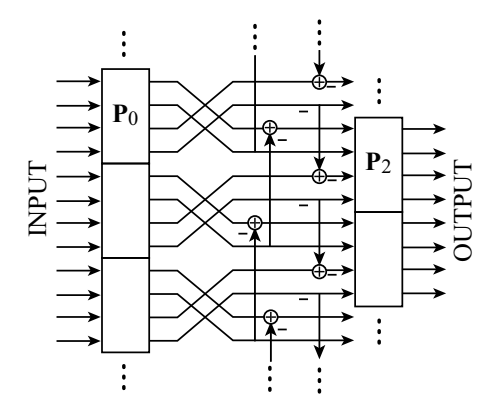

(b)
Figure 3: Time-domain filter bank structure (shown for $M=4$, analysis bank). (a) BOLPFB. (b) FOLPFB.

where

$$
\begin{aligned}
& \boldsymbol{\Lambda}_{T O}(z)=\left[\begin{array}{cc}
z^{-2} \hat{\boldsymbol{\Lambda}}_{T O}(z) & \mathbf{0}_{M / 2} \\
\mathbf{0}_{M / 2} & -z^{-1} \hat{\boldsymbol{\Lambda}}_{T O}\left(z^{-1}\right)
\end{array}\right] \\
& \hat{\boldsymbol{\Lambda}}_{T O}(z)=\left[\begin{array}{cccc}
1 & 0 & & \\
-p_{0} z^{-1} & 1 & 0 & \\
0 & -p_{1} z^{-1} & 1 & \ddots \\
& \ddots & \ddots & \ddots
\end{array}\right] \\
& \times\left[\begin{array}{cccc}
1 & u_{0} z & 0 & \\
0 & 1 & u_{1} z & \ddots \\
& 0 & 1 & \ddots \\
& & \ddots & \ddots
\end{array}\right] .
\end{aligned}
$$

This buillding block is third-order. Obviously if $p_{i}=0$, the obtained FB is a FOLPFB (also if $p_{i}=u_{i}=0$, the FB is a BOLPFB). The building block can yield wider range of filter lengths up to thirdorder. However, the third-order building block is a restricted version of the HOF ones. In the next subsection, we derive the structure of the HOF building blocks based on the concept of the third-order LPFBs.

\subsection{Structure of HOF Building Blocks}

Before we formulate a HOF building block, consider the form in (7). The differences between each block diagonal component in $\boldsymbol{\Lambda}_{T O}(z)$ are signs and powers of $z$. Furthermore, $\boldsymbol{\Lambda}_{T O}(z)$ maintains the lifting structures which imply that $\boldsymbol{\Lambda}_{T O}(z)$ belongs to a subclass of complete lifting matrices.

Generally an $M \times M$ nonsingular matrix B can always be factorized into $\mathbf{B}=\overline{\mathbf{P}} \overline{\mathbf{L}} \overline{\mathbf{D}} \overline{\mathbf{U}}$ where all matrices have the size $M \times M$ and $\overline{\mathbf{P}}$ is a permutation matrix, $\overline{\mathbf{L}}$ is a lower triangular matrix, $\overline{\mathbf{D}}$ is a diagonal matrix, and $\overline{\mathbf{U}}$ is an upper triangular matrix [7]. By using the LDU matrix factorization, we assume the delay matrix instead of $\boldsymbol{\Lambda}_{T O}(z)$ as

$$
\boldsymbol{\Lambda}_{H O F}(z)=\left[\begin{array}{cc}
\hat{\boldsymbol{\Lambda}}_{H O F}(z) & \mathbf{0} \\
\mathbf{0} & -\hat{\boldsymbol{\Lambda}}_{H O F}\left(z^{\prime}\right)
\end{array}\right]
$$

where $\hat{\boldsymbol{\Lambda}}_{H O F}(1)$ is an $M / 2 \times M / 2$ nonsingular matrix with the LDU factorization and $\hat{\Lambda}_{H O F}\left(z^{\prime}\right)$ has different powers of $z$ from $\hat{\boldsymbol{\Lambda}}_{H O F}(z)$. If $\mathbf{E}(z)$ has all filter lengths to be $K M$, the LP condition is represented as $[1,2]$

$$
\mathbf{E}(z)=\hat{\mathbf{D}} z^{K-1} \mathbf{E}\left(z^{-1}\right) \mathbf{J}
$$

where $\hat{\mathbf{D}}$ is the diagonal matrix with entries +1 or -1 , depending on whether the filter is symmetric $(+1)$ or antisymmetric $(-1)$. Under the assumption in (8), the condition can be simplified as

$$
\hat{\boldsymbol{\Lambda}}_{H O F}(z)=z^{-K+1} \hat{\Lambda}_{H O F}\left(z^{\prime-1}\right) .
$$

The condition in (10) implies that the powers of $z$ in $\hat{\Lambda}_{H O F}\left(z^{\prime}\right)$ depends on those in $\hat{\Lambda}_{H O F}(z)$.

Although the powers of $z$ in (10) could have various patterns, there are a few requirements for $z$ to obtain good frequency responses. First, all diagonal elements in $\hat{\Lambda}_{H O F}(z)$ should have the same powers. Second, the difference between the diagonal powers of $z$ in $\hat{\Lambda}_{H O F}(z)$ and $\hat{\Lambda}_{H O F}\left(z^{\prime}\right)$ is $|1|$. Third, powers of $z$ should be changed gradually to yield consecutive filter coefficients. For example, there should not be $z^{-3}$ next to $z^{-1}$. We decide the powers and positions of $z$ using these rules.

Another important rule is considered in this paper. That is, each element of $\hat{\boldsymbol{\Lambda}}_{\mathrm{HOF}}(z)$ corresponds to only one power of $z$. It avoids cumbersome calculations to obtain the inverse of a HOF building block. Furthermore, it provides a fact which can be considered $\hat{\boldsymbol{\Lambda}}_{H O F}(1)$ and powers of $z$ separately to yield the inverse. It contributes to decrease calculation costs.

Next, we reduce the number of redundant parameters in $\hat{\boldsymbol{\Lambda}}_{H O F}(1)$. It is commonly known that positions of each matrix in the LDU decomposition can be changed without any disadvantage [9]. In this paper we assume $\hat{\Lambda}_{H O F}(1)$ can be decomposed into

$$
\hat{\Lambda}_{H O F}(1)=\text { PDLU. }
$$

Furthermore, $\mathbf{P D}$ can be merged into the block diagonal $\operatorname{diag}\left(\mathbf{U}_{1}, \mathbf{V}_{1}\right)$ since

$$
\mathbf{W}^{\prime} \operatorname{diag}(\mathbf{P D}, \mathbf{P D})=\operatorname{diag}(\mathbf{P D}, \mathbf{P D}) \mathbf{W}^{\prime}
$$

is always true. Consequently, $\hat{\boldsymbol{\Lambda}}_{H O F}(1)=\mathbf{L U}$ for a HOF building block. Additionally, the structure of $\hat{\Lambda}_{H O F}(1)$ is restricted to obtain the same filter length in both banks. If we need an order- $N$ HOF building block, $\hat{\boldsymbol{\Lambda}}_{H O F}(1)$ is limited to be the block diagonal matrix whose maximum (and at least one) block size is $(N-1)$. The restriction turns out both $\mathbf{L}$ and $\mathbf{U}$ have to be the block diagonal matrices such as

$$
\begin{aligned}
\mathbf{L} & =\operatorname{diag}\left(\mathbf{L}_{0}, \mathbf{L}_{1}, \ldots\right) \\
\mathbf{U} & =\operatorname{diag}\left(\mathbf{U}_{0}, \mathbf{U}_{1}, \ldots\right),
\end{aligned}
$$

where $\mathbf{L}_{n}$ and $\mathbf{R}_{n}(n=0,1, \ldots)$ are $\tilde{N} \times \tilde{N}(\tilde{N} \leq(N-1))$ lower and upper triangular matrices whose all diagonal elements are 1 , respectively. Afterwards, we assume the size of $\mathbf{L}_{0}$ and $\mathbf{U}_{0}$ is $(N-$ $1) \times(N-1)$ for simplicity.

By combining (10) and the structure of $\hat{\boldsymbol{\Lambda}}_{H O F}(1)$, we obtain the complete structure of $\boldsymbol{\Lambda}_{H O F}(z)$ as follows:

$$
\boldsymbol{\Lambda}_{H O F}(z)=z^{-N} \operatorname{diag}\left(\hat{\boldsymbol{\Lambda}}_{H O F}\left(z_{i, j}\right),-\hat{\boldsymbol{\Lambda}}_{H O F}\left(z_{i, j}^{\prime}\right)\right)
$$

where $\hat{\boldsymbol{\Lambda}}_{H O F}(1)=\mathbf{L} \mathbf{U}$ and $s_{i, j}$ and $s_{i, j}^{\prime}$ are, respectively, the powers of $z_{i, j}$ and $z_{i, j}^{\prime}$ as

$$
s_{i, j}=\left\{\begin{array}{l}
s_{i, i}=-1 \\
s_{i, j+1}=s_{i, j}+1 \\
s_{i+1, j}=s_{i, j}-1
\end{array}, \quad s_{i, j}^{\prime}=-\left(s_{i, j}+1\right) .\right.
$$

Furthermore, $N$ is the highest positive power among $z_{i, j}$ and $z_{i, j}^{\prime}$, which is adopted for causal filters. Consequently, the entire HOF building block is

$$
\mathbf{G}_{H O F}(z)=\operatorname{diag}(\mathbf{Q}, \mathbf{V}) \mathbf{W}^{\prime} \boldsymbol{\Lambda}_{H O F}(z) \mathbf{W}
$$




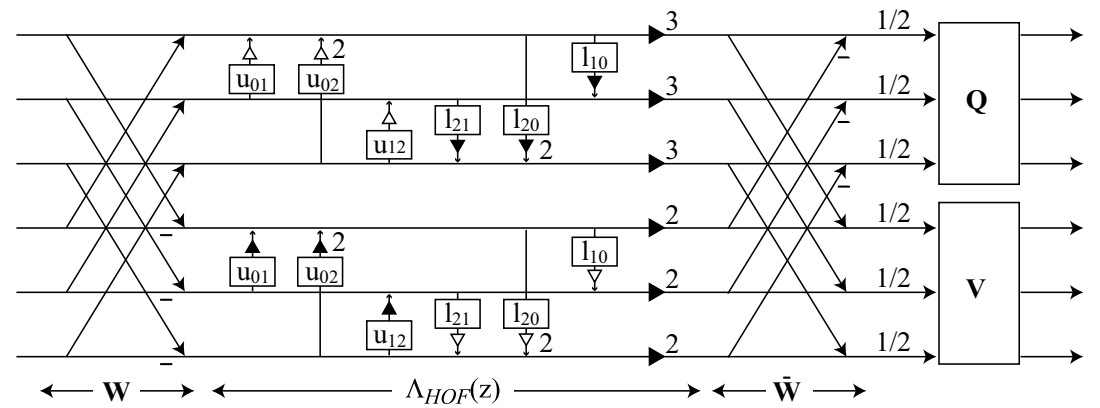

Figure 4: Structure of analysis HOF building block (shown for $M=6$ and fifth-order).
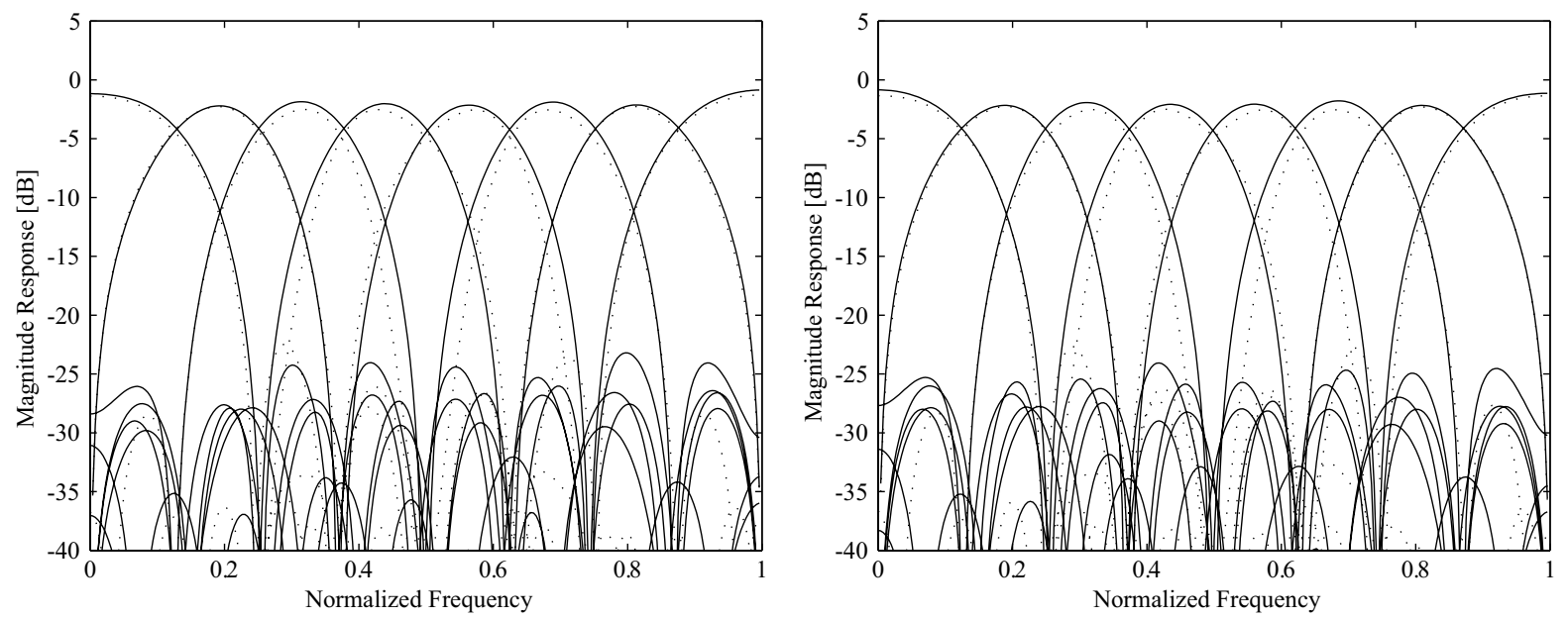

Figure 5: Normalized frequency responses of the proposed $8 \times 32$ third-order BOLPFB (solid lines) and the traditional $8 \times 32$ BOLPFB with the cascaded order-1 structure (dotted lines). (Left) analysis bank. (Right) synthesis bank.

and its inverse is

$$
\mathbf{G}_{H O F}^{-1}(z)=\mathbf{W} \mathbf{\Lambda}_{I H O F}(z) \mathbf{W}^{\prime T} \operatorname{diag}\left(\mathbf{Q}^{-1}, \mathbf{V}^{-1}\right) .
$$

$\Lambda_{I H O F}(z)$ is formulated as

$$
\boldsymbol{\Lambda}_{\text {IHOF }}(z)=z^{-N^{\prime}} \operatorname{diag}\left(\hat{\boldsymbol{\Lambda}}_{\text {IHOF }}\left(z_{i, j}^{\prime \prime}\right),-\hat{\boldsymbol{\Lambda}}_{\text {IHOF }}\left(z_{i, j}^{\prime}\right)\right)
$$

where $\mathbf{Q}$ and $\mathbf{V}$ are $M / 2 \times M / 2$ nonsingular matrices, $\hat{\boldsymbol{\Lambda}}_{I H O F}(1)=$ $\mathbf{U}^{-1} \mathbf{L}^{-1}, z_{i, j}^{\prime \prime}=s_{i, j}+2$ and $N^{\prime}$ is the highest positvie power among $z_{i, j}^{\prime \prime}$ and $z_{i, j}^{\prime}$. If $\mathbf{U}=\mathbf{L}=\mathbf{I}$, the HOF building block is the same as an order-1 building block. Furthermore, if $\mathbf{L}=\mathbf{I}$ and $(\mathbf{U}-\mathbf{I})$ is a block diagonal matrix with Jordan blocks, the building block is the same as a FOLPFBs' component. It means that these two traditional building blocks are special cases of the proposed HOF structure. The building block structure is illustrated in Fig. 4. In this figure, a black/white triangle indicates a delay/advance element and the number besides the triangle is the power of the element.

It can permit an odd-order (up to $(M-1)$-th order) LPFB with one HOF building block. Furthermore, the building block does not need to calculate its inverse as the matrix polynomial for $\mathbf{G}^{-1}(z)$ directly, since the inverse can be easily obtained as a product of each element of $\hat{\Lambda}_{I H O F}(1)$ and $z_{i, j}^{\prime \prime}$ or $z_{i, j}^{\prime}{ }^{1}$.

\subsection{Realization of Even-Order LPFBs with HOF building blocks}

In the above subsection, we formulate the HOF building block. However, only odd-order LPFBs are permitted by using a HOF

\footnotetext{
${ }^{1}$ e.g. $\hat{\boldsymbol{\Lambda}}_{I H O F}\left(z_{i, j}^{\prime \prime}\right)$ is the product of the $(i, j)$-th element of $\hat{\boldsymbol{\Lambda}}_{I H O F}(1)$ and $z_{i, j}^{\prime \prime}$.
}

building block. To realize even-order LPFBs is a challenging problem. Fortunately, the proposed building block $\mathbf{G}(z)$ satisfies the order- $N$ LP building block condition $\mathbf{G}(z)=z^{-N} \hat{\mathbf{D}} \mathbf{G}\left(z^{-1}\right) \hat{\mathbf{D}}$ [2]. It suggests that HOF building blocks can be cascaded each other without losing the LP property. The previous subsection also introduced an order-1 building block as a subclass of a HOF building block. After all, even-order LPFBs can be realized by cascading order-1 and HOF building blocks.

\subsection{Comparison of the Numbers of Required Building Blocks and Design Parameters}

First, we compare the number of building blocks (except $\mathbf{E}_{0}$ ) to the traditional BOLPFBs. By using the HOF structure, the number of the blocks can be at most two up to $M$-th order. In other words, the structure can have various filter lengths within two HOF building blocks. Conversely, the number of building blocks for traditional BOLPFBs depends on their required filter lengths. In the even-channel case, it is easily calculated as $(K-1)$.

Next, we describe the number of design parameters. The comparison from third to sixth-order is shown in Table 1. In this table, $L, m$ and $\lfloor\cdot\rfloor$ are $M / 2, M$ modulo 6 and a function that returns the largest integer less than or equal to $(\cdot)$, respectively. The number of design parameters of $\mathbf{G}_{H O F}(z)$ in (17) could be some patterns due to the structures of $\mathbf{L}$ and $\mathbf{U}$. However, the largest numbers are shown in the table for fair comparison. It is obvious that the HOF structure always has fewer parameters than the traditional cascaded order-1 structure.

Generally many design parameters yield better filter responses, however, the amount of the improvement and the eliminable number of parameters are trade-off. Especially if many channels or long 

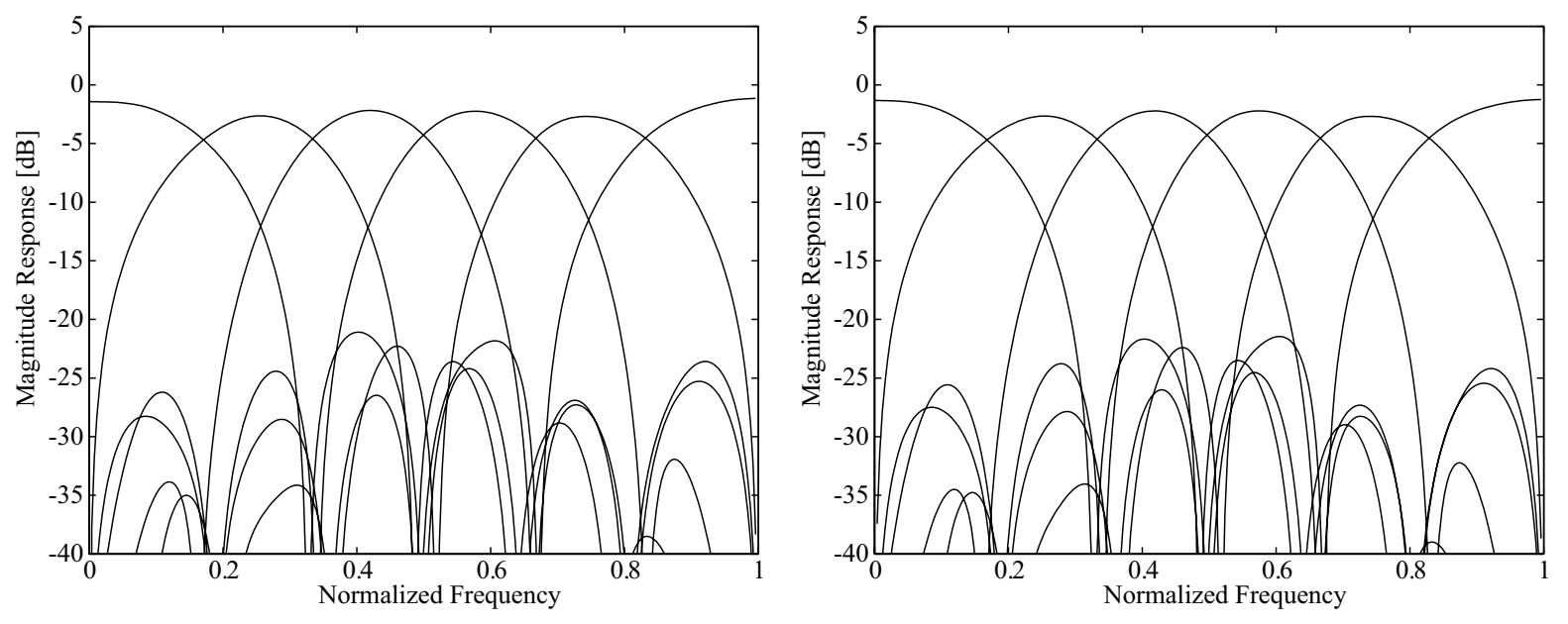

Figure 6: Normalized frequency responses of the proposed $6 \times 36$ fifth-order BOLPFB. (Left) analysis bank. (Right) synthesis bank.

Table 1: Comparison of the Number of Design Parameters

\begin{tabular}{c|c|c}
\hline Order & Cascaded Order-1 & HOF Build. blocks \\
\hline 3 & $3 L^{2}$ & $2\left(L^{2}+\lfloor L / 2\rfloor\right)$ \\
\hline 4 & $4 L^{2}$ & $3 L^{2}+2\lfloor L / 2\rfloor$ \\
\hline 5 & $5 L^{2}$ & $2\left(L^{2}+3\lfloor L / 3\rfloor+\lfloor m / 4\rfloor\right)$ \\
\hline 6 & $6 L^{2}$ & $3 L^{2}+2(3\lfloor L / 3\rfloor+\lfloor m / 4\rfloor)$ \\
\hline
\end{tabular}

filters are required, reduction of the number of design parameters is much recommended than slightly modification of filter performance.

\section{DESIGN}

In this section, we present two different designs of BOLPFBs with the HOF building blocks. All FBs are optimized to have good stopband attenuation which is formulated as [1]

$$
\begin{aligned}
& C_{\text {ana. st pb. }}=\sum_{i=0}^{M-1} \int_{\omega \in \text {-thstopband }}\left|H_{i}\left(e^{j \omega}\right)\right|^{2} d \omega \\
& C_{\text {syn. stpb. }}=\sum_{i=0}^{M-1} \int_{\omega \in \text { i-thstopband }}\left|F_{i}\left(e^{j \omega}\right)\right|^{2} d \omega .
\end{aligned}
$$

The design examples are listed as follows:

Design Example 1: 8-Channel 32-Tap (Third-Order)

This is an example of the structure in Section 3.1. The FB has one $\mathbf{G}_{H O F}(z)$. The normalized frequency responses are shown as solid lines in Fig. 5. To compare with the conventional method, the frequency responses of the $8 \times 32$ BOLPFB with order- 1 building blocks [2] are also depicted as dotted lines in the figure. Obviously, the proposed BOLPFB shows comparable results in both banks despite of fewer building blocks and design parameters. Furthermore, in objective performance, the worst stopband attenuations of the HOF structure and the order- 1 structure are $-23.20(\mathrm{~dB})$ and -22.47 $(\mathrm{dB})$, respectively.

Design Example 2: 6-Channel 36-Tap (Fifth-Order)

It has one $(M-1)$-th order $\mathbf{G}_{H O F}(z)$ which is described in Section 3.2. Fig. 6 depicts its frequency responses.

It is observed that all examples have good stopband attenuations in both banks. As previously mentioned, BOLPFBs with the HOF building blocks always have fewer building blocks and design parameters than the traditional one. It may lead to reduce implementation costs for not only design parameters but also a hardware architecture. These advantages are the reasons for choosing the HOF building blocks.

\section{CONCLUSIONS}

In this paper, we proposed a new structure of BOLPFBs by using HOF building blocks. The building block is useful to design longer filters. Furthermore, the number of building blocks is smaller than the traditional cascaded order- 1 structure which leads to reduce implementation costs in practical signal processing applications. Our future work is to derive more efficient structures.

\section{Acknowledgment}

This work was supported by the Ministry of Education, Science, Sports and Culture, Grant-in-Aid for JSPS Fellows.

\section{REFERENCES}

[1] G. Strang and T. Q. Nguyen, Wavelets and Filter Banks, Cambridge, MA: Wellesley-Cambridge, 1996.

[2] T. D. Tran, R. L. de Queiroz, and T. Q. Nguyen, "Linear phase perfect reconstruction filter bank: lattice structure, design, and application in image coding," IEEE Trans. Signal Process., vol. 48, pp. 133-147, Jan. 2000.

[3] A. Makur, A. Muthuvel, and P. V. Reddy, "Eigenstructure approach for complete characterization of linear-phase FIR perfect reconstruction analysis length $2 M$ filterbanks," IEEE Trans. Signal Process., vol. 52, pp. 1801-1804, June 2004.

[4] L. Gan and K.-K. Ma, "A simplified lattice factorization for linear-phase perfect reconstruction filter bank," IEEE Signal Process. Lett., vol. 8, pp. 207-209, July 2001.

[5] T. D. Tran, J. Liang, and C. Tu, "Lapped transform via timedomain pre- and post-filtering," IEEE Trans. on Signal Process., vol. 51, pp. 1557-1571, June 2003.

[6] A. Muthuvel and A. Makur, "Eigenstructure approach for characterization of FIR PR filterbanks with order one polyphase," IEEE Trans. Signal Process., vol. 49, pp. 2283 2291, Oct. 2001.

[7] G. H. Golub and C. F. V. Loan, Matrix Computations, 3rd Ed., Baltimore, MD: Johns Hopkins Univ. Press, 1996.

[8] I. Daubechies and W. Sweldens, "Factoring wavelet transforms into lifting steps," J. Fourier Anal. Applicant., vol. 4, pp. 247-269, 1998.

[9] T. D. Tran, " $M$-channel linear phase perfect reconstruction filter bank with rational coefficients," IEEE Trans. Circuits Systems I Fund. Theory Appl., vol. 49, pp. 914-927, Jul. 2002. 